\title{
HAPPY OR NOT? ROOTS OF STUDENT SATISFACTION IN CLASS SESSIONS
}

Matthew Metzgar ${ }^{1}$

\begin{abstract}
Businesses and organizations are turning to fast feedback devices that measure consumer satisfaction. These standalone devices often have four or five different smiley-face buttons that can quickly gauge consumer sentiment. These devices are becoming popular due to the short time commitment needed to provide feedback.

This instructor has piloted the use of a smiley-face survey tool that is used at the end of each class session. Initial results suggest the role of the instructor may play a lesser role in student happiness for a given class. External factors, such as outside event or incoming GPA, appear to have a larger effect on class satisfaction. Data from classes and sample email exchanges will be presented. This type of fast feedback system has many potential benefits and low startup costs.
\end{abstract}

Key words: student feedback, higher education, polling systems

\section{Introduction}

Businesses and organizations are turning to fast feedback devices that measure consumer satisfaction. These standalone devices often have four or five different smiley-face buttons that can quickly gauge consumer sentiment. Both for-profit and non-profit organizations have utilized these devices as a quick way to gain consumer feedback.

These feedback devices can be either physical devices or created in electronic form. Physical devices are being used at private establishments like restaurants and retails, and also in government organizations such as airports (Happy or Not Ltd. 2019). Physical devices are also used on college campuses for a variety of purposes.

Companies like Netflix also use these feedback systems in electronic form (Happy or Not Ltd. 2019). Electronic fast feedback systems can also be used by companies to rate customer service or overall experience with a website. Electronic systems can also be used internally to measure employee satisfaction.

Given the widespread use of such systems, the author began researching the potential use of a system in a classroom setting. Due to large class sizes, using a physical device seemed unwise. With hundreds of students attempting to leave a classroom out of one door at once, feedback would not be reliable. Therefore, the instructor decided to use an electronic version of the feedback system in class. 
This paper discusses the use of a smiley-face/emoticon survey tool that is used at the end of each class session to measure student satisfaction. This paper will highlight the instructor's results of the tool across different sections and across time. Initial results are very intriguing, suggesting the role of the instructor may play a lesser role in student happiness for a given class. Data from classes and sample email exchanges are presented.

\section{Literature Review}

Student feedback systems in higher education have been criticized for a variety of reasons (Alderman, Towers, and Bannah 2012). A common criticism is that the feedback questions lack validity and reliability. These criticisms and concerns then lead to misuse or apathy from faculty and administrators.

To remedy this, some authors recommend more in-depth types of feedback, such as focus groups and student interviews (King et al. 1999). Others view fast feedback as an effective way to gauge student knowledge (van den Berg, Ed 2003).

Another issue is the effectiveness of paper feedback versus electronic feedback (Ardalan et al. 2007). In their paper Ardalan et al. (2007) show that paper and electronic feedback provide roughly the same level of quality in regards to student feedback. Building on this, more continuous-type feedback has been studied under electronic feedback system (Soreanu and Saucan 2003). The scalability and cost-effectiveness of electronic versus paper feedback have led to a general move in that direction by Universities, including the author's own University.

Hatziapostolou and Paraskakis (2010) did use a multi-faceted system that used emoticons among other tools. The system was implemented for two years and designed to provide quality feedback to students. The authors ultimately concluded that the system strongly affected student motivation.

Another recent research effort looked at using emoticons for mathematics tasks (D'Agostin 2014). This study involved using emoticons with a task. The authors found that the emoticon feedback system was effective in revealing students' emotions towards the task.

This also illustrates the issue of how frequent should feedback be taken. A recent study (Surgenor 2013) shows some of the potential problems with summative feedback. With traditional course evaluations, instructors may not receive feedback until a course is over. With feedback sections each class, there is more opportunity for an instructor to gather, reflect, and act on student feedback.

Student feedback has the potential for positive or negative changes in instructor practices. A recent study (Flodén 2017) shows student feedback pushes teaching into a more helpful direction for students. However, the same study showed there can be a danger of professors pandering to student demands. Therefore, students have to be judicious in how they alter their teaching in connection to student feedback.

\section{Experiment}

This experiment took place in a large, public Southern University. The trial classes were an upper-level required business course, Managerial Economics. These classes were relatively large, consisting of 100 or 200 students per class. The feedback question was given via PollEverywhere. PollEverywhere was available to all students at no cost as it is provided by the University. The instructor has taught this course for a number of years and has also used various classroom feedback systems for many years.

The instructor began using the feedback scale in the spring semester of 2019 . The question was simply worded, "How was today's class?" and was presented at the end of each class. Students would respond by choosing one of the five smiley faces to rep- 
resent their satisfaction level. There were no in-between choices offered, as students could only select one of the five options.

Based on the literature review, the intent was to present a simple, subjective question to students. One question only was chosen for simplicity, time savings, and to create holistic responses.

Students were given one point for answering the question, regardless of what option they chose. Student feedback was not anonymous, but it did take some instructor effort to match student's names with their response.

The reliability of students' responses was obviously a concern. Students received full credit regardless of which response they chose. There is of course the possibility that students could choose responses randomly or choose opposite responses on purpose. These concerns will be further discussed below.

This feedback question at the end of class was meant to bookend the attendance question given at the beginning of class. An attendance question was posted within the first minute or two of class to encourage students to arrive on time and be ready to participate. The feedback question at the end of class was designed to wrap up class and allow student feedback. Since this feedback question was worth a point, it is assumed that most if not all of students present in the classroom regularly responded to this question.

Students chose on of the five following smiley faces for their feedback:
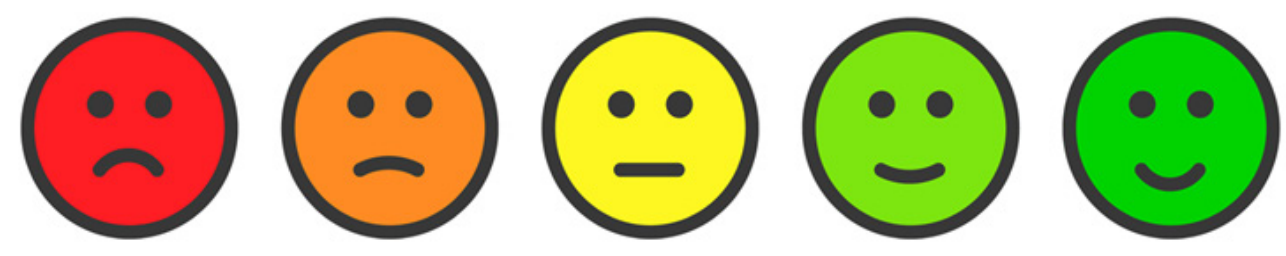

Figure 1: Emoticon options

From left to right, the instructor interpreted these results as: "highly unsatisfied", "unsatisfied", "neutral", "satisfied", or "highly satisfied".

One potential disadvantage with this methodology is that the polling question, "how was today's class?" may then include many non-academic factors. For example, if a student was tired or overstressed, they may report having a negative class experience. Rephrasing the question in other ways, such as "was the instructor effective today?" may give provide better feedback to the instructor.

\section{Results}

From the very first feedback question, the majority of student responses were either "satisfied" or "highly satisfied". Here is a sample output from a class with 197 registered students: 


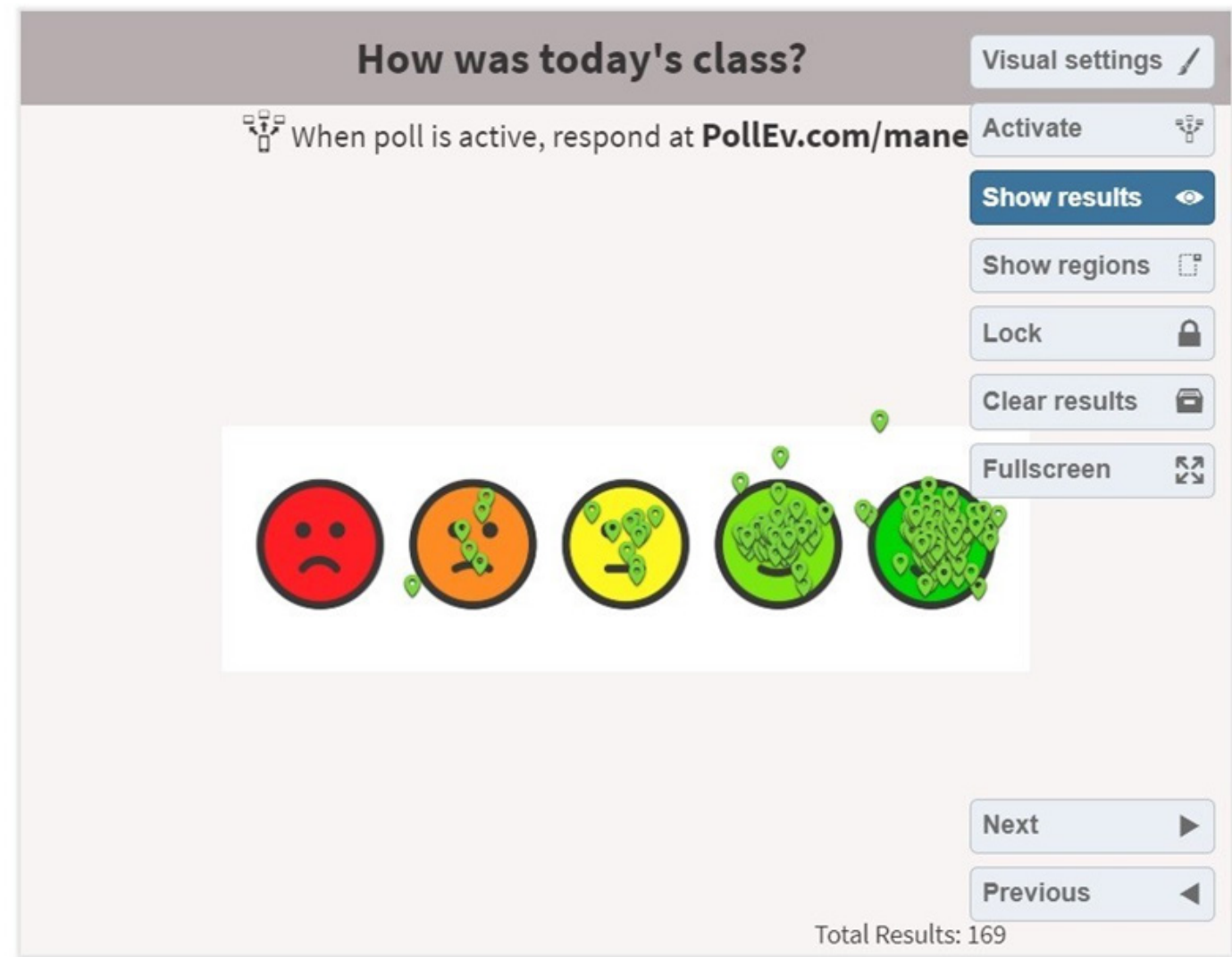

Figure 2: Sample Results

The first finding is that the survey results were not random. Survey results across different sections were often very consistent. Therefore, it appears that most students were attempting to be forthright with their response, though this may not have been the case for all students.

The sample results show the results were overwhelming positive. This was obviously very gratifying from an instructional standpoint. Part of the reason for this is that the instructor has taught this course many times each semester for a number of years. This accumulated experience has produced a polished course for students that have improved over time.

The large number of positive responses also made the small number of students with negative responses easier to identify. Conversely, the small number of negative responses also made any type of detailed quantitative analysis difficult. Most of the negative responses came early in the semester, and some classes had no negative responses at all.

In terms of averages over time, Table 1 presents the average percentage for each category aggregated over the course of a semester for one section of the course with 197 registered students.

Table 1: Average Results Over One Semester

\begin{tabular}{|c|c|}
\hline Response & Average Percentage (\%) \\
\hline Highly Unsatisfied & 0.3 \\
\hline Unsatisfied & 1.2 \\
\hline Neutral & 5.8 \\
\hline Satisfied & 20.3 \\
\hline
\end{tabular}




\begin{tabular}{|c|c|}
\hline Response & Average Percentage (\%) \\
\hline Highly Satisfied & 72.4 \\
\hline
\end{tabular}

Source: Author's Data

Overall, the average results parallel the results from a sample class shown earlier. The majority of students felt satisfied or highly satisfied with each class over the course of the semester.

\section{Discussion}

While the majority of students were satisfied, some students were not. Early in the semester, the instructor identified and reached out to those students who were unsatisfied with class. These students were then studied in a non-quantitative manner, as once they were contacted by the instructor their future survey results were no longer random.

In talking with those students and also reviewing their academic records, four distinct themes emerged. These themes are now discussed below.

1. Students with negative responses often had lower than average GPAs.

The average GPA for a student enrolled in this class was 3.10. For students that gave an unsatisfied or highly unsatisfied response early in the semester, their average GPA was 2.83. This may show that these groups of students may be struggling with other classes as well.

This difference in average GPA mirrors other data trends the instructor has previously uncovered. For those students entering this class with a GPA less than 3.0, previous internal analysis has shown they have a much lesser chance of passing the class. For those entering with a GPA of less than 2.5, the odds of success are extremely low.

2. Students with negative responses often then did poorly on the exams.

Exam scores were compared between all students and any student who submitted an unsatisfied or highly unsatisfied response before the first exam. The overall class average on Exam \#1 was 79\%. The average for those students giving unsatisfied/highly unsatisfied responses was $73 \%$.

Students then are clearly self-identifying problems with the course material early in the semester. These problems then come to light with the exam results. This ability to self-identify could be a positive tool if an effective early remediation program could help those students before the first exam.

The correlation between the first and second trend are also evident. Students entering the course with lower than average GPAs tend to encounter early difficulties. These difficulties become exposed on the first exam. The end result is a correlation between incoming GPA and odds of passing the course.

3. Students with negative responses would often cite their difficulty in understanding the material, not in the instructional approach.

Upon starting this experiment, the instructor was concerned that this could be a tool for students to "vent" or place blame on the instructor. This turned out not to be the case. The vast majority of students who further described their lack of satisfaction focused on the material, not the instructor.

Students would describe how they felt the instruction was sufficient, but that they had trouble fully understanding and absorbing the material. In some ways, this was comforting for the instructor as the explanation of the material seemed sufficient. However, this group of students was not able to achieve full understanding of the material.

4. Students gave negative responses for reasons unrelated to the class.

A number of students, when contacted, would cite outside reasons for a negative response. This could involve the weather, stress from other class, or work. Sometimes students would respond saying that just had low energy on a given day. 


\section{Further Issues}

The study of negative responders was primarily non-quantitative in nature due to a potential number of measurement errors. First, there could be a sample bias in those who responded to the instructor's email. Perhaps students who did have issues with the instructional approach were reluctant to respond. They may have worried about grade retribution or other harm.

Second, after the instructor responded to those with unsatisfactory/highly unsatisfactory responses, those students were less likely to respond in the same way in the future. It's likely that students would feel like they were simply repeating the same issues. Therefore, the number of negative student responses declined over the semester in a non-random way.

Third, there is also the possibility were purposely non-accurate in their responses or were choosing their responses randomly. Given that students would receive one point no matter what their response, there was no grade incentive to reveal their most accurate response.

\section{Conclusion}

This initial pilot study gave students an opportunity to voice feedback on a classper-class basis. Such a system could have many potential benefits. It could provide early feedback for the instructor on the effectiveness of his or her approach.

In this experiment, student feedback often identified those students who struggled with material. The student feedback was primarily about the difficulty of the material, not the instructional approach. Other students also voiced negative feedback for outside concerns, such as weather, work, academic stress, etc.

One immediate use of this feedback system is that it identifies students who are more likely to perform poorly on exams. However, this information is also predicted by students' lower incoming GPA. In this experiment, there was a correlation between students providing negative feedback and students with a lower incoming GPA.

From a practical standpoint, this type of feedback system has many benefits and few drawbacks. It is easy to administer, especially if the institution already has a polling system installed across campus. This system can also be enacted with minimal faculty training.

Future research could take many different directions. Comparisons could be made across small versus large classes. Various pre-existing student characteristics could also be analyzed to see if there is any relationship between incoming student characteristics and satisfaction level.

From an academic standpoint, this paper adds to the existing literature on how student perceive the class experience. The results of this experiment show that students are often dissatisfied with day-to-day class experience due to difficulty with the material and outside events. Problems with the material may be due to pre-existing knowledge deficits, as students with lower incoming GPAs tend to report more dissatisfaction.

Overall, this pilot experiment was a valuable way to gauge student feedback with a novel smiley face system. Such a system mimics the fast feedback displays used by businesses and other organizations. The instructor continues to use this system in class at the present. There are many potential benefits from fast student feedback, and it is a relatively easy system to administer. The instructor hopes to gain further future insights that can ultimately increase student success.

\section{References}

ALDERMAN, LYN, STEPHEN TOWERS, AND SYLVIA BANNAH. "Student feedback systems in higher educa- 
tion: A focused literature review and environmental scan." Quality in Higher Education 18, no. 3 (2012): 261-280.

ARDALAN, ALI, ROYA ARDALAN, SAMUEL COPPAGE, AND WILLIAM CROUCH. "A comparison of student feedback obtained through paper-based and web-based surveys of faculty teaching." British Journal of Educational Technology 38, no. 6 (2007): 1085-1101.

D'AGOSTIN, FABIO. "The Value of Emoticons in Investigating Student Emotions Related to Mathematics Task Negotiation." Mathematics Education Research Group of Australasia (2014).

FLODÉN, JONAS. "The impact of student feedback on teaching in higher education." Assessment \& Evaluation in Higher Education 42.7 (2017): 1054-1068.

Happy or Not Ltd. 2019. "Customer Stories." Accessed August 3, 2019. https://www.happy-or-not.com/en/customer-stories/

HATZIAPOSTOLOU, THANOS, AND IRAKLIS PARASKAKIS. "Enhancing the impact of formative feedback on student learning through an online feedback system." Electronic Journal of e-Learning 8, no. 2 (2010): 111-122.

KING, MALCOLM, IAN MORISON, GARY REED, AND GRAZYNA STACHOW. "Student feedback systems in the business school: a departmental model." Quality assurance in Education 7, no. 2 (1999): 90-100.

SOREANU, PETER, AND EMIL SAUCAN. "Semi-continuous monitoring of student feedback in interactive synchronous e-learning environments." In Proceedings 3rd IEEE International Conference on Advanced Technologies, pp. 276-277. IEEE, 2003.

SURGENOR, PAUL WG. "Obstacles and opportunities: addressing the growing pains of summative student evaluation of teaching." Assessment \& Evaluation in Higher Education 38, no. 3 (2013): 363-376.

VAN DEN BERG, ED. "Teaching, learning, and quick feedback methods." Australian Science Teachers Journal 49, no. 2 (2003): 28. 